\title{
MINIMAL INJECTIVE RESOLUTIONS UNDER FLAT BASE CHANGE
}

\author{
HANS-BJØRN FOXBY ${ }^{1}$ AND ANDERS THORUP
}

\begin{abstract}
ABSTRACr. For a flat morphism $\varphi: A \rightarrow B$ of noetherian rings, the minimal injective resolution of the $B$-module $M \otimes_{A} B$ is described in terms of the minimal injective resolution of the finitely generated $A$-module $M$ and the minimal injective resolutions of the fibers of $\varphi$.
\end{abstract}

Throughout this note $A$ and $B$ denote commutative noetherian rings with multiplicative identities. For an $A$-module $M$, a prime ideal $\mathfrak{p}$ in $A$, and an integer $i$ the (cardinal) number $\mu_{A}^{i}(\mathfrak{p}, M)$ denotes the dimension of $\operatorname{Ext}_{A}^{i}(A / \mathfrak{p}, M)_{\mathfrak{p}}=\operatorname{Ext}_{A_{\mathfrak{p}}}^{i}\left(k(\mathfrak{p}), M_{\mathfrak{p}}\right)$ considered as a vector-space over the field $k(\mathfrak{p})=A_{\mathfrak{p}} / \mathfrak{p} A_{\mathfrak{p}}$. Now let

$$
0 \rightarrow M \rightarrow I^{0} \rightarrow I^{1} \rightarrow \cdots \rightarrow I^{i} \rightarrow \ldots
$$

be a minimal injective resolution of $M$. Then $\mu_{A}^{i}(\mathfrak{p}, M)=\mu_{A}^{0}\left(\mathfrak{p}, I^{i}\right)$ and this is the number of copies of the injective hull $E_{A}(A / \mathfrak{p})$ of $A / \mathfrak{p}$ in the decomposition of $I^{i}$ into indecomposable injective modules. For this fact, and for other facts concerning the numbers $\mu_{A}^{i}(\mathfrak{p}, M)$ and minimal injective resolutions, consult Bass' paper [1] (in particular, §2).

Now let $\varphi: A \rightarrow B$ be a ringhomomorphism making $B$ into a flat $A$-module, and let $M$ be a finitely generated $A$-module. The main result of this note describes the minimal injective resolution of the $B$-module $M \otimes_{A} B$ by expressing the number $\mu_{B}^{n}\left(q, M \otimes_{A} B\right.$ ) (for each $n$ and each prime ideal $q$ in $B)$ in terms of the numbers $\mu_{A}^{q}(q \cap A, M)$ and $\mu_{C}^{p}(q C, C)$ where $C$ is the fiber of $\varphi$ at $q \cap A$. All the numbers here are finite, since $M$ is finitely generated.

THEOREM. Let $\varphi: A \rightarrow B$ be a flat ringhomomorphism, let a be a prime ideal in $B$, and let $C$ denote the ring $B /(q \cap A) B$ with the prime ideal $q^{\prime}=q C$. Then for a finitely generated $A$-module $M$ and for all numbers $n$ there is an equality of numbers

$$
\mu_{B}^{n}\left(\mathfrak{q}, M \otimes_{A} B\right)=\sum_{p+q=n .} \mu_{C}^{p}\left(\mathfrak{q}^{\prime}, C\right) \mu_{A}^{q}(\mathfrak{q} \cap A, M) .
$$

This result will be proved below, but first we mention two immediate

Received by the editors September 21, 1976.

AMS (MOS) subject classifications (1970). Primary 13C10, 13D05; Secondary 13H10.

Key words and phrases. Minimal injective resolution, $\mu_{A}^{n}(\mathfrak{p}, M)$, flat base change, injective dimension, Gorenstein ring, quasi-isomorphism.

${ }^{1}$ Supported, in part, by the Danish Natural Science Research Council. 
applications to the injective dimensions of the $A$-module $M$ and the $B$-module $M \otimes_{A} B$. These dimensions are here denoted by $\operatorname{id}_{A} M$ and $\operatorname{id}_{B}\left(M \otimes_{A} B\right)$ respectively (and they might be infinite).

Corollary 1. Let $A$ and $B$ be local rings and let $\varphi: A \rightarrow B$ be a flat local ringhomomorphism. If $M$ is a finitely generated nonzero $A$-module, then

$$
\mathrm{id}_{B}\left(M \otimes_{A} B\right)=\mathrm{id}_{A} M+\mathrm{id}_{C} C
$$

where $C=B / \mathrm{m} B$ and $\mathrm{m}$ is the maximal ideal in $A$.

In particular, $\operatorname{id}_{B}\left(M \otimes_{A} B\right)$ is finite if and only if $\mathrm{id}_{A} M$ is finite and $C$ is a Gorenstein ring.

Proof. Corollary 1 follows directly from the Theorem since $\operatorname{id}_{A} M=$ $\sup \left\{i \mid \mu_{A}^{i}(\mathrm{~m}, M) \neq 0\right\}$ (and similarly for $\operatorname{id}_{B}\left(M \otimes_{A} B\right.$ ) and $\operatorname{id}_{C} C$ ) (cf. $[1,(3.2)$ Corollary]).

REMARK 1. The main result of [4] is a result in the same direction as Corollary 1. It states that if $\varphi: A \rightarrow B$ is a flat ringhomomorphism and if $E$ is an injective $A$-module, then

$$
\operatorname{id}_{B}\left(E \otimes_{A} B\right)=\sup \left\{\operatorname{id}_{F(\mathfrak{p})} F(\mathfrak{p}) \mid \mathfrak{p} \in \operatorname{Ass}_{A} E\right\}
$$

where $F(\mathfrak{p})=k(\mathfrak{p}) \otimes_{A} B$ is the fiber at $\mathfrak{p}$.

Suppose that $A$ is local with maximal ideal $\mathrm{m}$ and let $\hat{A}$ denote the completion of $A$ (in the m-adic topology). If there exists a Gorenstein module over $A$ (that is, a finitely generated module $G$ with depth $G=\operatorname{id} G<\infty$ ), then all the fibers of $A \rightarrow \hat{A}$ are Gorenstein rings (cf. [9, (2.8) Theorem] and consult [8] and [3] for facts about Gorenstein modules). The next result generalizes this to arbitrary finitely generated modules of finite injective dimension.

COROllary 2. Let $M$ be a finitely generated nonzero module over the local ring $A$. If $\operatorname{id}_{A} M<\infty$, then the formal fiber $C=\hat{A} \otimes_{A} k(\mathfrak{p})$ at $\mathfrak{p}$ is a Gorenstein ring for all $\mathfrak{p}$ in the support of $M$.

Proof (of Corollary 2). The local rings of $C$ are of the form $C_{\mathrm{a} C}$ where $a$ is a prime ideal in $\hat{A}$ lying over $\mathfrak{p}$. Now apply Corollary 1 to the homomorphism $A_{\mathfrak{p}} \rightarrow \hat{A_{\mathrm{q}}}$ using the fact that the $\hat{A_{\mathrm{q}}}$-module $M_{\mathfrak{p}} \otimes_{A_{\mathfrak{p}}} \hat{A_{\mathrm{q}}}=\hat{M_{\mathrm{q}}}$ is of finite injective dimension.

EXAMPLE. Ferrand and Raynaud have constructed a domain of dimension one such that the generic formal fiber $\hat{A} \otimes_{A} Q$ is not a Gorenstein ring ( $Q$ is the field of fractions), cf. [2]. In particular, this $\operatorname{ring} A$ has no Gorenstein modules, and Corollary 2 shows even more: If the finitely generated module $M$ over this $\operatorname{ring} A$ is of finite injective dimension, then $M$ is artinian.

A third application of the Theorem can be found in [5]. Namely: If $M$ is a finitely generated $A$-module and $\mathfrak{p} \subseteq$ q are two prime ideals, then $\mu_{A}^{i}(\mathfrak{p}, M) \leqslant$ $\mu_{A}^{i+l}(\mathfrak{q}, M)$ for all $i$, where $l=\operatorname{dim}\left(A_{\mathrm{q}} / \mathrm{p} A_{\mathrm{q}}\right)$.

Proof of The Theorem. Since the $\mu^{i}$ is unchanged under localization we may place ourself in the following situation: $\mathfrak{p}$ and $\mathfrak{a}$ are maximal ideals in, 
respectively, $A$ and $B$ such that $\varphi(\mathfrak{p}) \subseteq \mathrm{q}$. We are then required to prove

$$
\mu_{B}^{n}\left(q, M \otimes_{A} B\right)=\sum_{p+q=n} \mu_{C}^{p}(q C, C) \mu_{A}^{q}(\mathfrak{p}, M)
$$

for all $n$, when $C=B / p B$.

To prove this we shall for any $A$-module $M$ construct $C$-linear isomorphisms

$$
\operatorname{Ext}_{B}^{n}\left(l, M \otimes_{A} B\right) \simeq \coprod_{p+q=n} \operatorname{Ext}_{C}^{p}(l, C) \otimes_{k} \operatorname{Ext}_{A}^{q}(k, M)
$$

where $k=A / \mathfrak{p}$ and $l=B / q=C / q C$. Then for $M$ finitely generated we get the desired formula by counting dimensions over $l$.

During the proof we work in the category of complexes of modules over the different rings considered. Recall that a morphism $\alpha: X \rightarrow Y$ between complexes of modules over a ring $R$ is called a quasi-isomorphism if it induces isomorphisms $H^{i}(\alpha): H^{i}(X) \stackrel{\sim}{\rightarrow} H^{i}(Y)$ on the cohomology modules for all $i$. This property of a morphism is preserved by any of the functors

$$
\operatorname{Hom}_{R}(P,-), \operatorname{Hom}_{R}(-, I), \text { and }-\otimes_{R} M,
$$

where $P$ is a bounded above complex of $R$-projective modules, $I$ is a bounded below complex of $R$-injective modules and $M$ is an $R$-flat module, cf. [6, Chapter I, Lemma 6.2, p. 64].

To construct the isomorphisms in (1) we choose a minimal $A$-injective resolution $I$ of $M$ and a quasi-isomorphism $I \otimes_{A} B \rightarrow J$ where $J$ is a complex of $B$-injective modules with $J^{i}=0$ for $i<0$, cf. [6, Chapter I, Lemma 4.6, p. 42]. Choose, furthermore, a $C$-projective resolution $P$ of $l$. Then we have quasi-isomorphisms

$$
M \rightarrow I, \quad I \otimes_{A} B \rightarrow J, \text { and } P \rightarrow l .
$$

The isomorphisms in (1) are now established by defining quasi-isomorphisms $\alpha^{*}$ and $\beta_{*}$

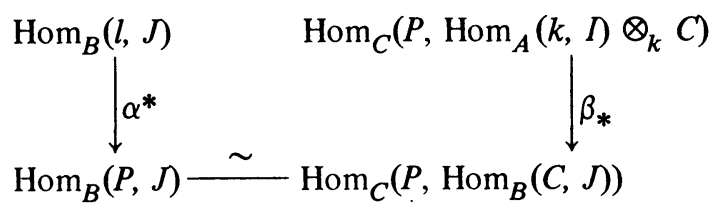

and identifying the two sides of (1) with the cohomology of the two sides of (2). Here $\alpha^{*}$ is induced by the quasi-isomorphism $\alpha: P \rightarrow l$ and since $J$ is a complex of $B$-injective modules we see that $\alpha^{*}$ is a quasi-isomorphism. And $\beta_{*}$ is induced by the composite $C$-linear morphism $\beta$ defined by the commutative diagram




To prove that $\beta$ is a quasi-isomorphism we choose a resolution $F$ of $k$ by finitely generated $A$-free modules. Then we have a quasi-isomorphism $F \rightarrow k$ and a commutative diagram of complexes of $B$-modules

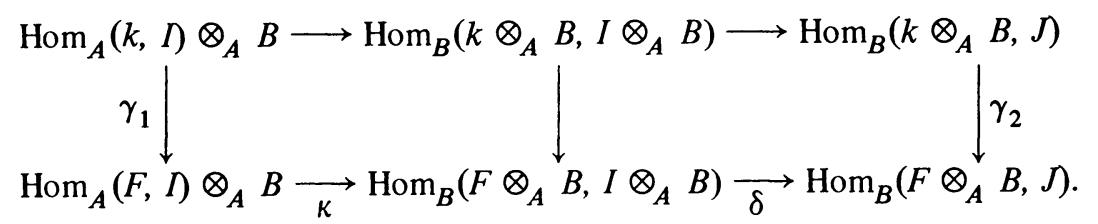

The morphism $\gamma_{1}$ is a quasi-isomorphism since $I$ consists of $A$-injective modules and $B$ is $A$-flat. The morphism $\kappa$ is an isomorphism since each $F^{i}$ is finitely generated $A$-free. The morphism $\delta$ is a quasi-isomorphism since $F \otimes_{A} B$ consists of $B$-free modules and $I \otimes_{A} B \rightarrow J$ is a quasi-isomorphism. The morphism $\gamma_{2}$ is a quasi-isomorphism since $J$ consists of $B$-injective modules and $F \otimes_{A} B \rightarrow k \otimes_{A} B$ is a quasi-isomorphism by flatness of $B$. As $\beta$ is a quasi-isomorphism and $P$ a complex of $C$-projective modules it follows that $\beta_{*}$ is a quasi-isomorphism.

Passing to cohomology we see by flatness of $B$ that $M \otimes_{A} B \rightarrow I \otimes_{A} B$ is a quasi-isomorphism and, hence, the composite $M \otimes_{A} B \rightarrow I \otimes_{A} B \rightarrow J$ is a quasi-isomorphism, i.e. $J$ is a $B$-injective resolution of $M \otimes_{A} B$. Consequently,

$$
H^{n}\left[\operatorname{Hom}_{B}(l, J)\right]=\operatorname{Ext}_{B}^{n}\left(l, M \otimes_{A} B\right)
$$

To compute the cohomology of $\operatorname{Hom}_{C}\left(P, \operatorname{Hom}_{A}(k, I) \otimes_{k} C\right)$, we remark, that since $I$ is a minimal resolution, the differentials in $\operatorname{Hom}_{A}(k, I)$ are zero. Then this holds for $\operatorname{Hom}_{A}(k, I) \otimes_{k} C$ as well and so the differentials in $\operatorname{Hom}_{C}\left(P, \operatorname{Hom}_{A}(k, I) \otimes_{k} C\right)$ are induced from $P$ only. And we get for the cohomology

$$
\begin{aligned}
H^{n}\left[\operatorname{Hom}_{C}\right. & \left.\left(P, \operatorname{Hom}_{A}(k, I) \otimes_{k} C\right)\right] \\
& =\prod_{p+q=n} H^{P}\left[\operatorname{Hom}_{C}\left(P, \operatorname{Hom}_{A}\left(k, I^{q}\right) \otimes_{k} C\right)\right] \\
& =\prod_{p+1=n} \operatorname{Ext}_{C}^{p}\left(l, \operatorname{Hom}_{A}\left(k, I^{q}\right) \otimes_{k} C\right) \\
& =\prod_{p=q=n} \operatorname{Ext}_{C}^{p}(l, C) \otimes_{k} \operatorname{Hom}_{A}\left(k, I^{q}\right) \\
& =\prod_{p+q=n} \operatorname{Ext}_{C}^{p}(l, C) \otimes_{k} \operatorname{Ext}_{A}^{q}(k, M) .
\end{aligned}
$$

REMARK 2. The proof of the quasi-isomorphisms in (2) works for any base change situation

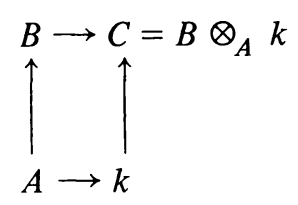


where $A \rightarrow B$ is a flat homomorphism of noetherian rings and $A \rightarrow k$ is a (module-) finite ringhomomorphism and $l$ is any finitely generated $C$-module. In the derived category $D(C)(2)$ becomes an isomorphism

$$
\mathbf{R H o m}_{B}\left(l, M \otimes_{A} B\right) \simeq \operatorname{RHom}_{C}\left(l, \mathbf{R H o m}_{A}(k, M) \otimes_{k} C\right)
$$

(in the notation of [6]). However, the calculation of the cohomology of the right-hand side of $\left(2^{\prime}\right)$ made in the end of the proof requires some extra conditions on the ring $k$, such as being a field.

REMARK 3. Corresponding to (2) we have also a spectral sequence:

$$
E_{2}^{p q}=\operatorname{Ext}_{C}^{p}\left(l, \operatorname{Ext}_{A}^{q}(k, M) \otimes_{k} C\right) \Rightarrow \operatorname{Ext}_{B}^{n}\left(l, M \otimes_{A} B\right) .
$$

In the situation described at the beginning of the proof of the Theorem this spectral sequence gives the inequality

$$
\mu_{B}^{n}\left(q, M \otimes_{A} B\right) \leqslant \sum_{p+q=n} \mu_{C}^{p}(q C, C) \mu_{A}^{q}(\mathfrak{p}, M) .
$$

This inequality has been studied in some special cases by Paugam in [7]. The equality (0) shows that all the differentials $E_{r}^{p q} \rightarrow E_{r}^{p+r, q-r+1}$ are zero (for $r \geqslant 2$ ).

\section{BIBLIOGRAPHY}

1. H. Bass, On the ubiquity of Gorenstein rings, Math. Z. 82 (1963), 8-28. MR 27 \#3669.

2. D. Ferrand and M. Raynaud, Fibres formelles d'un anneau local noethérien, Ann. Sci. École Norm. Sup. (4) 3 (1970), 295-311. MR 42 \#7660.

3. H.-B. Foxby, Gorenstein modules and related modules, Math. Scand. 31 (1972), 267-284. MR 48 \#6094.

4. __ Injective modules under flat base change, Proc. Amer. Math. Soc. 50 (1975), 23-27.

5. _ On the $\mu^{i}$ in a minimal injective resolution. II, Københavns Univ. Mat. Inst. (preprint).

6. R. Hartshorne, Residues and duality, Lecture Notes in Math., vol.20, Springer-Verlag, Berlin and New York, 1966. MR 36 \#5145.

7. M. Paugam, Comportement des $\mu_{i}$ de Bass dans les extensions plates, C.R. Acad. Sci. Paris 280 (1975), A1267-A1270. MR 51 \# 10324.

8. R.Y. Sharp, Gorenstein modules, Math. Z. 115 (1970), 117-139. MR 41 \#8401.

9. $\quad$ The Euler characteristic of a finitely generated module of finite injective dimension, Math. Z. 130 (1973), 79-93. MR 47 \#8518.

KøbenhaVns Universitets Matematiske Institut, Universitetsparken 5, DK 2100 KøBENHAVN $\varnothing$, DaNMARK 